\title{
How to safely extubate a patient in the emergency department: a user's guide to critical care
}

\author{
Sara H. Gray, BSc, MD, MPH*; John A. Ross, $\mathrm{MD}^{\dagger}$; Robert S. Green, BSc, MD, DABEM ${ }^{\ddagger}$
}

\section{CLINICAL SCENARIO}

A 35-year-old male is found unconscious at the bottom of a staircase. His Glasgow Coma Scale (GCS) score is 3, and he is intubated by the paramedics for failure to protect his airway. On assessment in the emergency department (ED), the GCS score remains 3, but his examination and investigations (including a normal head computed tomographic [CT] scan) are unremarkable except for a serum ethanol of $97 \mathrm{mmol} / \mathrm{L}$. Five hours later, he remains intubated in the ED but is starting to wake up. Should you extubate him or sedate him to keep him intubated? How do you ensure the optimal outcome for this patient?

\section{INTRODUCTION}

The rate of critically ill patients presenting to the ED is rising, and ED lengths of stay for these patients are increasing. ${ }^{1-4}$ Some patients remain in the ED long enough to improve clinically, and ED physicians are often faced with the clinical decision described above.

One recent article described safely extubating trauma patients in the ED who have improved clinically. ${ }^{5}$ However, there are no published algorithms or guidelines for assessing patients for possible extubation in the ED. It is not certain that rules for extubation should be any different in the ED than elsewhere in the hsopital.

Keywords: emergency department, extubation, patient safety
We developed the algorithm in Figure 1 for extubating adult patients based on expert opinion, evidence from other care settings, and our clinical experience. This represents the criteria that we use for extubation.

\section{EXTUBATION IN THE ED}

When patients are assessed for possible intubation, we routinely assess three domains: failure to oxygenate and ventilate, failure to maintain an airway, and anticipated clinical course. To evaluate patients for extubation in the ED, these three domains should be assessed and found to be acceptable: patients must be able to oxygenate and ventilate and maintain a patent airway, and their anticipated clinical course must be consistent with extubation (see Figure 1). The overriding concept is that the pathophysiologic state that mandated intubation has been addressed and resolved.

\section{Which patients should be considered?}

\section{Step 1: there is resolution of the process necessitating intubation}

Although many patients may fall into this category, likely candidates in the ED would include intoxicated or overdose patients in whom the intoxication has resolved and minor head injury patients who are improving clinically (likely with a negative head CT scan and significant trauma ruled out). Extubation may also be considered for pulmonary edema patients who are significantly improved.

From the *Faculty of Medicine, University of Toronto, and Department of Emergency Medicine and the Interdepartmental Division of Critical Care, St. Michael's Hospital and the Li Ka Shing Knowledge Institute, Toronto, ON; †Department of Emergency Medicine, Dalhousie University, Halifax, NS, and AIME Airway Course; $¥$ Division of Critical Care Medicine, Department of Anesthesia, and Department of Emergency Medicine, Dalhousie University, Halifax, NS.

Correspondence to: Dr. Sara H. Gray, 30 Bond Street, Room 4-036 Queen, Toronto, ON M5B 1W8; grays@smh.ca.

This article has been peer reviewed.

(c) Canadian Association of Emergency Physicians

DOI 10.2310/8000.2012.120795 


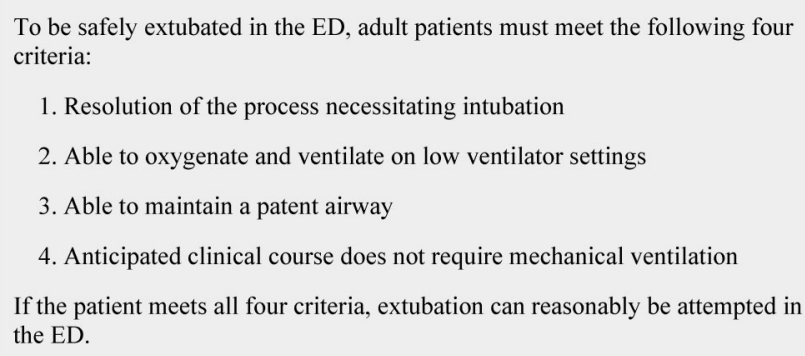

Figure 1. Safe extubation algorithm. ED = emergency department.

There are also a small number of patients who are intubated and on further inquiry are found to have declined aggressive life support or to be resuscitated. These patients are obvious candidates for ED extubation, and in most cases, extubation should proceed despite the ongoing disease process.

\section{Step 2: the patient is able to oxygenate and ventilate on low ventilator settings}

Low ventilator settings include the following:

- Spontaneous ventilation with low support (pressure support $\leq 10$ in most cases)

- Positive end-expiratory pressure (PEEP) $\leq 8$

- Tidal volume (TV) $\geq 5 \mathrm{cc} / \mathrm{kg}$

- Negative inspiratory force (NIF) or maximum inspiratory pressure $\geq-30 \mathrm{~cm} \mathrm{H}_{2} \mathrm{O}$ (normal -90 to $-120 \mathrm{~cm} \mathrm{H}_{2} \mathrm{O}$ )

The ability to oxygenate and ventilate can be assessed by an arterial blood gas (ABG) and oxygen saturation readings. Patients should have an acceptable arterial oxygen tension $(>70)$ and oxygen saturation $(>92 \%)$ in most cases. Ideally, the fraction of inspired oxygen $\left(\mathrm{FiO}_{2}\right)$ should be 0.40 or less to ensure that the patient will be able to oxygenate without the ventilator.

For a patient on higher ventilator settings, consider a trial of spontaneous breathing (Figure 2).

\section{Step 3: the patient is able to maintain a patent airway}

- Patient is easily reintubated. To meet this criterion, the patient's laryngoscopy and intubation should have been easy initially, and the patient should have no known swelling or obstruction of the glottis or upper airway. Physicians should ensure that they clearly document the ease of intubation or that this information is obtained from emergency medical

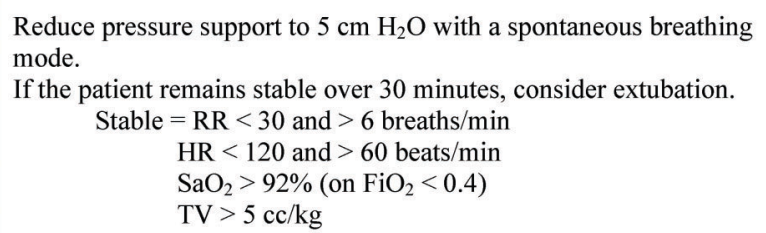

Figure 2. Spontaneous breathing trial. $\mathrm{FiO}_{2}=$ fraction of inspired oxygen; $\mathrm{HR}=$ heart rate; $\mathrm{RR}=$ respiratory rate; $\mathrm{SaO}_{2}$ $=$ saturated oxygen; $\mathrm{TV}=$ tidal volume

services (EMS) if the patient was intubated in the field.

- The patient is able to follow commands. Candidates for extubation should be able to open their eyes and track an object visually. They should be able to grasp their examiner's hand, stick out their tongue, and lift their head off the bed.

- The patient has a cuff leak. A cuff leak is an indirect way of indicating that there is no significant swelling at the glottis. It can be measured during volumecycled ventilation. First, there should be an audible cuff leak during positive pressure inhalation when the endotracheal tube (ETT) cuff is deflated. Second, this leak should comprise at least $25 \%$ of the inspired volume (e.g., $600 \mathrm{cc}$ in; at most $450 \mathrm{cc}$ measured out [exhaled TV], which indicates a leak of $150 \mathrm{cc}$ ). All ventilators display exhaled TV.

\section{Step 4: anticipated course does not require mechanical ventilation}

This step requires the emergency physician's judgment, which includes confidence in and comfort with the procedure of extubation (see below). Removing the ventilator necessitates that the patient is able to assume the workload associated with breathing, in terms of both managing secretions and managing the cardiac and respiratory workloads associated with breathing independently. Accordingly, the patient should have few secretions (with a decent cough) and be hemodynamically stable (without the need for vasopressor medications).

The criteria for hemodynamic stability are as follows:

- Oxygen saturation $>92 \%\left(\right.$ on $\left.\mathrm{FiO}_{2}<0.4\right)$

- Heart rate $<100$ beats/min

- Respiratory rate $<30 / \mathrm{min}$

- Systolic blood pressure $>90 \mathrm{~mm} \mathrm{Hg}$

- No active cardiac ischemia 
Lastly, there should be no other mitigating factor that would preclude safe extubation. Possible factors would include cervical spine injury or instability, acute lung injury, acute pharyngeal injury, or plans for an imminent operation or transport. Most importantly, attending physicians must take the time to carefully consider each case and ensure that they feel comfortable proceeding with extubation. If the attending physician feels concerned or uncomfortable, extubation should not be pursued in the ED. These criteria form a general guideline; however, individual EDs may want to develop their own extubation criteria "checklist" based on their unique resources and patient profiles.

\section{CLINICAL SCENARIO MANAGEMENT}

A 35-year-old male is found unconscious at the bottom of a staircase. His GCS score is 3, and he is intubated by the paramedics for failure to protect his airway. On assessment in the ED, his GCS score remains 3, but his examination and investigations are unremarkable except for a serum ethanol of $97 \mathrm{mmol} / \mathrm{L}$. Five hours later, he remains intubated but is starting to wake up in your ED. You decide to assess him for possible extubation.

Step 1: resolution of the process necessitating intubation

The patient was intubated because of alcohol intoxication resulting in a decreased level of consciousness and an inability to protect his airway. He has now awoken, and it seems that the alcohol that he ingested has lost its effect.

\section{Step 2: the patient is able to oxygenate and ventilate on low ventilator settings}

$\mathrm{He}$ is on minimal ventilator settings (pressure support ventilation 10, $\mathrm{FiO}_{2}$ 0.3, $\mathrm{PEEP}$ 5), with an $\mathrm{O}_{2}$ saturation of $97 \%$. He has a normal chest radiograph and a normal respiratory rate. An ABG reading has not been done, but you have no reason to suspect lung disease or hypoventilation.

\section{Step 3: the patient is able to maintain a patent airway}

He was an easy intubation and now is wide awake, moving spontaneously. He is able to follow commands and lift his head off the bed. It is confirmed that he has an adequate cuff leak. Although he was initially intubated for failure to protect his airway, this indication had now clearly resolved.

\section{Step 4: anticipated course does not require mechanical ventilation}

The patient remains hemodynamically stable, without any sign of active ischemia. His vital signs are normal, and his respirations are easy. Indeed, his lungs seem quite normal. No further investigations or operations are planned that might require ventilation or airway protection. Accordingly, the patient has met our four criteria for possible extubation.

The basic extubation procedure is as follows:

1. Explain the procedure to the patient; position the patient on his or her side or sitting upright.

2. Obtain assistance from the nurse, respiratory therapist, or both.

3. Prepare a high-flow oxygen mask and have a full airway cart nearby.

4. Using rigid suction, remove upper airway secretions.

5. Hold the ETT in place and remove the ETT ties.

6. At end inspiration, deflate the cuff and quickly and smoothly remove the ETT during exhalation.

7. Place a high-flow oxygen mask and monitor the patient closely for 30 minutes minimum.

The physician safely removes the ETT. The patient remains hemodynamically stable, is able to cough well, and has an oxygen saturation of $95 \%$ on room air. The patient is spared a prolonged intubation and an unnecessary admission for an intoxication that has now resolved.

\section{CONCLUSIONS}

ED patients require intubation for various reasons, some of which are of transient duration. Extubation in the ED should be approached in a logical manner, with resolution of the disease process requiring intubation and with consideration of the general indications for intubation. In all cases, clinicians should consider the issues carefully and remain cautious. Stay within your comfort zone, but the use of this algorithm (see Figure 1) should allow more informed decisions about possible extubation in the ED. A detailed version of the algorithm is supplied for reference in Appendix 1. 
Competing interests: None declared.

Keywords: emergency department, extubation, patient safety

\section{REFERENCES}

1. Herring A, Wilper A, Himmelstein D, et al. Increasing length of stay among adult visits to US emergency departments, 2001-2005. Acad Emerg Med 2009;16:609-16, doi:10.1111/ j.1553-2712.2009.00428.x.

2. Lambe S, Washington D, Fink A, et al. Trends in the use and capacity of California's emergency departments 1990-1999. Ann Emerg Med 2002;39:389-6, doi:10.1067/mem.2002. 122433.

3. McCaig LF, Burt CW. National Hospital Ambulatory Medical Care Survey: 2002 emergency department summary. Adv Data 2004;(340):1-34.

4. Green RS, MacIntyre JK. Critical care in the emergency department: an assessment of the length of stay and invasive procedures performed on critically ill ED patients. Scand 7 Trauma Resusc Emerg Med 2009;17:47, doi:10.1186/17577241-17-47.

5. Weingart SD, Menaker J, Truong H, et al. Trauma patients can be safely extubated in the emergency department. $\mathcal{F}$ Emerg Med 2001;40:235-9, doi:10.1016/j.jemermed.2009.05.033.

\section{APPENDIX 1: DETAILED SAFE EXTUBATION ALGORITHM}

To be safely extubated in the emergency department (ED), adult patients must meet the following four criteria:

1. Resolution of the process necessitating intubation

- The original airway or breathing problem has resolved

2. Able to oxygenate and ventilate on low ventilator settings

- Options for assessing ability to oxygenate and ventilate would include oxygen saturation $>92 \%$ or a normal arterial blood gas. A common assessment of respiratory strength would be a negative inspiratory force (NIF) $>-30 \mathrm{~cm} \mathrm{H}_{2} \mathrm{O}$ (normal NIF values would be -90 to $-120 \mathrm{~cm} \mathrm{H}_{2} \mathrm{O}$ )

- Typical low ventilator settings would include the following:
- Pressure support $\leq 10$

- Positive end-expiratory pressure $\leq 8$

- Tidal volumes $\geq 5 \mathrm{~mL} / \mathrm{kg}$

- Fraction of inspired oxygen $\left(\mathrm{FiO}_{2}\right) \leq 0.40$

3. Able to maintain a patent airway

- Anatomically easy airway for laryngoscopy and intubation

- Patient follows commands (opens eyes, grasps hand, lifts head off bed).

- A cuff leak is present: with the cuff of the endotracheal tube deflated, a leak $>25 \%$ should be present (exhaled volume less than inhaled volume due to leak)

4. Anticipated clinical course does not require mechanical ventilation

a. Good cough with acceptable secretions

b. Hemodynamically stable, with no vasopressors

- Saturated oxygen $>92 \%$ on $\mathrm{FiO}_{2}<0.4$

- Heart rate $<100$ beats/min

- Respiratory rate $<30$ breaths/min

- Systolic blood pressure $>90 \mathrm{~mm} \mathrm{Hg}$

- No active cardiac ischemia

c. No other mitigating factor

- Cervical spine injury or instability

- Acute lung injury

- Acute pharygeal injury

- Plan for imminent operation or transport

- Attending physician feels uncomfortable with extubation

If the patient meets all four criteria, extubation can reasonably be attempted in the ED.

These criteria form a general guideline; however, individual EDs may want to develop their own extubation criteria "checklist" based on their unique resources and patient profiles. 